\title{
Experimenting Adaptive Services in Sea-Cloud Innovation Environment
}

\author{
Jingguo $\mathrm{Ge}^{1}$, Yulei $\mathrm{Wu}^{1}$, Tong $\mathrm{Li}^{1}$, Yuepeng $\mathrm{E}^{1}$, Chi Zhang ${ }^{2}$ \\ ${ }^{1}$ Computer Network Information Center, Chinese Academy of Sciences, Beijing, 100190, China \\ ${ }^{2}$ School of Information Science and Technology, University of Science and Technology of China, Anhui, 230026, China \\ Email: \{gejingguo, wuyulei, tongl, eyp\}cstnet.cn, chizhang@ustc.edu.cn
}

\begin{abstract}
Most of existing network testbeds can only support the experimentation of $L 2 \sim L 4$ forwarding protocols, leaving the evaluation of $\mathbf{L} 4 \sim \mathbf{L} 7$ applications still a tremendous challenge. This paper pioneers to present the design of sea-cloud innovation environment (SCIE) based on the software defined networking (SDN) and network functions virtualization (NFV) paradigms to support adaptive service-oriented experimentation, where the virtualized network functions (VNFs) can be implemented or deimplemented dynamically on network devices according to ondemand requirements. The experimentation is running to form an adaptive chain of network functions, which can be achieved by the protocol oblivious forwarding (POF) via user-defined fields and generic flow instruction set to forward the data to appropriate devices with VNFs. In SCIE, we demonstrate the experimentation of DPI service with on-demand requirement of security check.
\end{abstract}

Index Terms-Testbed, SDN, NFV, POF, adaptive serviceoriented experimentation

\section{INTRODUCTION}

The existing network testbeds, e.g., GENI and ToMaTo, can offer researchers the possibility to experiment the novel designs of future Internet architectures or protocols under realistic conditions. However, these network testbeds can only support the experimentation of innovative L2 L4 forwarding protocols, leaving the deployment and evaluation of upper layer services, e.g., virtual private LAN service (VPLS), stateful firewall, deep packet inspection (DPI), or other L4 L7 applications, still a tremendous challenge. To bridge this gap, we present a new network testbed, called sea-cloud innovation environment (SCIE), to support the experimentation of both forwarding protocols and service-oriented applications. In particular, this paper makes the following contributions:

- The principle of experimenting adaptive services in network testbed is proposed based on the integration of network functions virtualization (NFV) and software defined networking (SDN) paradigms [1]. The adaptive service-oriented experimentation can be supported by the protocol oblivious forwarding (POF) [2] via user-defined fields and generic flow instruction set in two aspects: 1) the virtualized network functions (VNFs) can be dynamically implemented or de-implemented on network devices to form an adaptive chain of functions to provide network services and 2) additional VNFs can be adaptively implemented according to the on-demand requirements of experimentation during its running, which can alter the existing function chain and adapt for new service requirements.

- A wide-area SCIE with hardware resources located at six cities of China is developed to realize the proposed principle for adaptive service-oriented experimentation with key technologies of heterogeneous resource description, resource virtualization and slicing, request description and visual rendering, and holographic measurement.

- The implementation of DPI service with on-demand request of security check is demonstrated to validate the effectiveness of proposed SCIE.

\section{THE PRINCIPLE OF ADAPTIVE SERVICE-ORIENTED EXPERIMENTATION ENABLED TESTBED}

To support the adaptive service-oriented experimentation, the user-defined fields associated with a set of flow instructions need to be enabled in the testbed. The POF is an emerging and promising technology to make the forwarding plane totally protocol-oblivious. With POF, the user-defined fields are free to be added or deleted during the running of experimentation. Therefore, the testbed can easily alter the paths of data flows and adapt for on-demand requirements of the experimentation.

The principle of adaptive service-oriented experimentation is to leverage the characteristics of decoupling functionality from location of NFV and dynamically deploy or un-deploy the VNFs at network devices upon on-demand requirements to form an adaptive chain of functions to provide network services. The SDN protocol is adopted to install flow entries on network devices based on requirements and configure the path of data flows [3]. In this case, the user-defined fields provided by POF can be exploited to support the services whose required fields are unknown.

On the other hand, additional VNFs can be adaptively deployed according to the on-demand requirements of experimentation during its running. This can alter the existing chain of functions and adapt for new service requirements. Under such a circumstance, the user-defined fields can be utilized to support network services and configuring the path of data flows in new function chain according to the field recognized by the underlying flow instruction set.

\section{SEA-ClOUd INNOVATION ENVIRONMENT (SCIE)}

To realize the proposed principle for adaptive serviceoriented experimentation, we developed a wide-area SCIE using 12 hardware racks with computation, networking and storage resources located geographically at six cities including Beijing, Shanghai, Shenyang, Wuxi, Hefei and Guangzhou in 
China, as shown in Fig. 1. The key technologies adopted in SCIE can be summarized as follows:

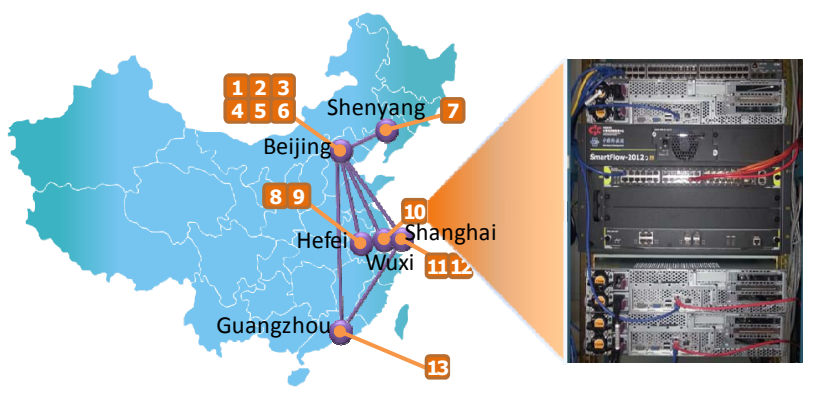

Fig. 1. The 12 hardware rack resources of SCIE and their geographical implementation in six cities in China

- Heterogeneous resource description and dynamic management. The SCIE adopts NDL-OWL to describe the distribution, request and assignment of heterogeneous resources, and uses SDN architecture for dynamic resource management and real-time monitoring.

- Resource virtualization. The SCIE uses both slice-based and time-based virtualization to handle sliceable and unsliceable resources, respectively. A sliced resource can be requested and created for each experimentation via web portal. The principle proposed in Section II can be instantiated at a sliced resource, as shown in Fig. 2.

- Holographic measurement. To decrease the impact on network performance, the SCIE mainly leverages passive measurements, and still provides active measurements to design the holographic measurement system.

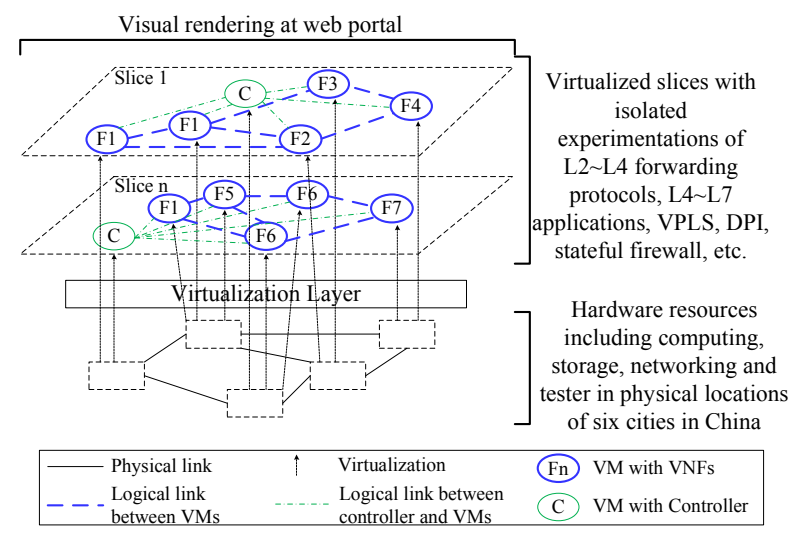

Fig. 2. The SCIE architecture

\section{DEMONSTRATION}

To evaluate the effectiveness of SCIE, we demonstrate the DPI service with on-demand requirement of security check while the experimentation is running.

For demonstration, seven VMs with corresponding storage and networking resources are requested at the SCIE for slice creation (see Fig. 3). One VM is utilized to implement the controller, four VMs (D1 D4) for DPI VNF, one VM (D6) for securing VNF, and the last one (D5) leaves for free. All VMs are implemented with POF. The dashed lines indicate the packet forwarding directions.
The DPI VNFs are pre-implemented in devices D1 D4 with POF enabled to provide the DPI service. The flow entries with dpi field to support DPI service can be installed to those devices by the controller. The data flows matching a set of entries on devices D1 D4 form a chain of functions, i.e., F1F2-F3-F4, as shown in Fig. 3. During the running of experimentation, the DPI VNFs should be adaptively deployed in device D5 by controller, due to the observation that D2 and D3 with DPI VNFs are over-loaded shown as the red dashed line in Fig. 3. The resulting chains of functions are F1-F2-F3F4 and F1-F5-F4.

If the on-demand requirements such as security check service launched by Security VNF are proposed during the experimentation running, the Security VNF is adaptively deployed on device D6, and the new sec field can be added to satisfy this request. By recognizing the sec field, the flow instruction set of POF can guide the data flows to device D6 from D3 and D5 for security checking without the assistant of controllers. The chain of functions is adaptively serviceoriented because it is changing due to the implementation of Security VNF with on-demand requirements. The resulting chains of functions become F1-F2-F3-F6-F3-F4 and F1-F5-F6F5-F4, as shown in Fig. 3.

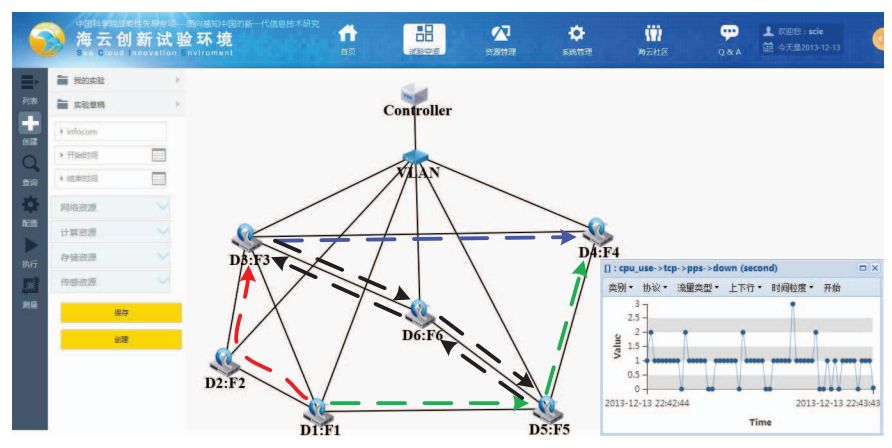

Fig. 3. The adaptive service-oriented experimentation shown on the web portal of SCIE

\section{CONCLUSIONS}

This paper has proposed the design principle of networking testbed for adaptive service-oriented experimentation, and developed a new wide-area sea-cloud innovation environment (SCIE) to validate its effectiveness via demonstration.

\section{ACKNOWLEDGMENT}

This work is partially supported by the Strategic Priority Research Program of the Chinese Academy of Sciences under XDA06010306, the 973 Program under 2012CB315803, and the NFSC under 61303241.

\section{REFERENCES}

[1] "Service-Aware Network Architecture Based on SDN, NFV, and Network Intelligence," White Paper, Intel ${ }^{\circledR}$ Architecture Processors.

[2] H. Song, "Protocol-Oblivious Forwarding: Unleash the Power of SDN through A Future-Proof Forwarding Plane," in Proc. of ACM HotSDN'13, pp. 127-132, 2013.

[3] S. Barkai, R. Katz, D. Farinacci and D. Meyer, "Software Defined FlowMapping for Scaling Virtualized Network Functions," in Proc. of ACM HotSDN'13, pp. 149-150, 2013. 